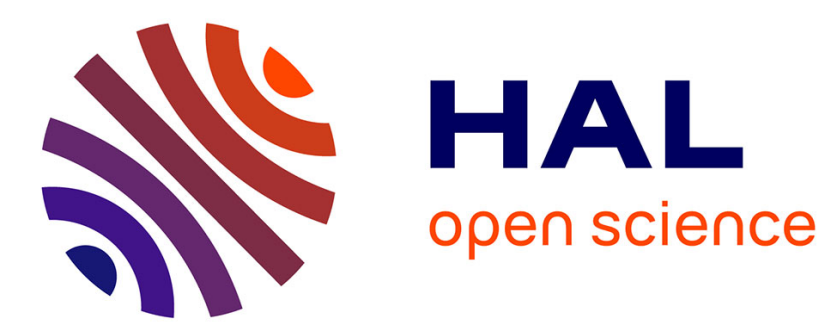

\title{
A Reinforcement Learning Approach to Interval Constraint Propagation
}

Frédéric Goualard, Christophe Jermann

\section{To cite this version:}

Frédéric Goualard, Christophe Jermann. A Reinforcement Learning Approach to Interval Constraint Propagation. Constraints, 2008, 13 (1-2), p.206-226. 10.1007/s10601-007-9027-7 . hal-00421423

\section{HAL Id: hal-00421423 \\ https://hal.science/hal-00421423}

Submitted on 2 Oct 2009

HAL is a multi-disciplinary open access archive for the deposit and dissemination of scientific research documents, whether they are published or not. The documents may come from teaching and research institutions in France or abroad, or from public or private research centers.
L'archive ouverte pluridisciplinaire HAL, est destinée au dépôt et à la diffusion de documents scientifiques de niveau recherche, publiés ou non, émanant des établissements d'enseignement et de recherche français ou étrangers, des laboratoires publics ou privés. 


\title{
A Reinforcement Learning Approach to Interval Constraint Propagation
}

\author{
Frédéric Goualard and Christophe Jermann \\ LINA, CNRS FRE 2729 - University of Nantes - France \\ 2, rue de la Houssinière - BP 92208 - F-44322 Nantes cedex 3 \\ \{Frederic.Goualard|Christophe. Jermann\}@univ-nantes.fr
}

\begin{abstract}
When solving systems of nonlinear equations with interval constraint methods, it has often been observed that many calls to contracting operators do not participate actively to the reduction of the search space. Attempts to statically select a subset of efficient contracting operators fail to offer reliable performance speed-ups. By embedding the recency-weighted average Reinforcement Learning method into a constraint propagation algorithm to dynamically learn the best operators, we show that it is possible to obtain robust algorithms with reliable performances on a range of sparse problems. Using a simple heuristic to compute initial weights, we also achieve significant performance speed-ups for dense problems.
\end{abstract}

\section{Introduction}

We consider the problem of finding tight enclosures for all the solutions of systems of nonlinear real equations ${ }^{1}$ :

$$
\begin{gathered}
f_{1}\left(x_{1}, \ldots, x_{n}\right)=0 \\
\ddots \\
f_{n}\left(x_{1}, \ldots, x_{n}\right)=0
\end{gathered}
$$

A successful approach associates interval domains to all variables and uses interval arithmetic [20] in a combination of contracting operators - to tighten the domains of the variables while retaining all solutions - and of an exploration algorithm that recursively splits domains.

An effective implementation of contracting operators relies on interval firstorder methods, which start from the initial domains and then solve each of the $n$ unary equations (projections):

$$
f_{i}\left(\boldsymbol{I}_{\mathbf{1}}, \ldots, \boldsymbol{I}_{\boldsymbol{i - 1}}, x_{i}, \boldsymbol{I}_{\boldsymbol{i}+\mathbf{1}}, \ldots, \boldsymbol{I}_{\boldsymbol{n}}\right)=0
$$

\footnotetext{
${ }^{1}$ In the following, we will refer to Syst. (1) even when considering systems in which all variables do not occur in all equations (sparse systems).
} 
in turn, where $\boldsymbol{I}_{\boldsymbol{j}}$ is the current interval domain for $x_{j}$. This process is iterated over all $f_{i}$ s until a fixed-point is reached (or, until the domains computed for the variables do not change too much).

For the linear case, the speed of convergence towards a solution depends heavily on the initial order of equations and variables, which defines the transver$s a l$, that is, the set of $n$ projections $\left(f_{i}, x_{i}\right)$ considered in Eq. (2). A classical result states that equations and variables should be initially reordered so as to make the corresponding coefficient matrix strictly diagonal dominant [23].

For the nonlinear case, it has been observed that nonlinear first-order methods are equally sensitive to the initial order of equations and variables that defines the $n$ projections considered. However, to our knowledge, there is no sure-fire static method to select projections that ensures prompt convergence. What is more, it appears $[9,11,8]$ that selecting more than $n$ projections may sometime speed the solving process up.

The bc3 algorithm [4] studied in this paper associates the good principles of first-order methods with a smart propagation algorithm devised by Mackworth [19] to ensure consistency in a network of relations. It also uses clever numerical methods to reduce the computational burden of solving projections.

Contrary to standard first-order methods, bc3 considers all $n^{2}$ possible projections instead of only $n$ of them, thereby avoiding a bad choice of a transversal. For sparse problems (those for which some variables do not occur in all equations), this is a reasonable strategy, since the number $p$ of unary equations to solve is of the order of $n$. On the other hand, for large dense problems (i.e., $n^{2} \gg n$ and $p \approx n^{2}$ ), the number of univariate equations to solve makes this approach computationally too expensive. In addition, depending on the problems, many projections may never lead to any tightening of the domains of the variables, for reasons that are not clearly understood yet. To make things worse, there may exist subsets of efficient projections, but only transiently at some point in the computation process. Therefore, there is no point in trying to statically select a subset of projections to consider in bc3.

In this paper, we embed the recency-weighted average [26] Reinforcement Learning method into bc3 to dynamically select the most efficient projections (that is, the ones leading to the maximum tightening of variables' domains). We experimentally show that the resulting algorithm outperforms bc3 for problems where no static transversal exists. We also present an heuristic to initialize weights associated to projections that leads to significant speed-ups with respect to bc3 when considering large dense problems with a static transversal.

In order to be reasonably self-content, we sketch the principles of interval constraint algorithms and we show the limits and weaknesses of bc3 in Section 2; We present in Section 3 how to add the recency-weighted average (rwa) Reinforcement Learning method to bc3 to address its shortcomings, and we describe the resulting algorithm, after having discussed how to fix the various parameters arising from the use of rwa. Our new algorithm is compared with bc3 on a set of standard problems in Section 4. Lastly, we compare our approach with previous related works in Section 5, and we outline directions for future researches in Section 6. 


\section{Interval Constraint Solving}

Classical iterative numerical methods suffer from defects such as loss of solutions, absence of convergence, and convergence to unwanted attractors due to the use of but a very small subset of the real numbers on computers: floating-point numbers [13] (aka floats). At the end of the fifties, Moore [20] popularized the use of intervals to control the errors made while computing with floats.

Interval arithmetic replaces floating-point numbers by closed connected sets of the form $\boldsymbol{I}=[\underline{\boldsymbol{I}}, \overline{\boldsymbol{I}}]=\{a \in \mathbb{R} \mid \underline{\boldsymbol{I}} \leqslant a \leqslant \overline{\boldsymbol{I}}\}$ from the set $\mathbb{I}$ of intervals, where $\underline{\boldsymbol{I}}$ and $\overline{\boldsymbol{I}}$ are floating-point numbers. In addition, each $n$-ary real function $\phi$ with domain $\mathcal{D}_{\phi}$ is extended to an interval function $\Phi$ with domain $\mathcal{D}_{\Phi}$ in such a way that the containment principle is verified:

$$
\forall A \in \mathcal{D}_{\phi} \forall \boldsymbol{I} \in \mathcal{D}_{\Phi}: A \in \boldsymbol{I} \Longrightarrow \phi(A) \in \Phi(\boldsymbol{I})
$$

Example 1 The natural interval extensions of addition and multiplication are defined by:

$$
\begin{aligned}
& \boldsymbol{I}_{1}+\boldsymbol{I}_{2}=\left[\underline{\boldsymbol{I}_{1}}+\underline{\boldsymbol{I}_{2}}, \overline{\boldsymbol{I}_{1}}+\overline{\boldsymbol{I}_{2}}\right] \\
& \boldsymbol{I}_{1} \times \boldsymbol{I}_{2}=\left[\min \left(\underline{\boldsymbol{I}_{1} \boldsymbol{I}_{2}}, \underline{\boldsymbol{I}_{1}} \overline{\boldsymbol{I}_{2}}, \overline{\boldsymbol{I}_{1}} \underline{\boldsymbol{I}_{2}}, \overline{\boldsymbol{I}_{1} \boldsymbol{I}_{2}}\right), \max \left(\underline{\boldsymbol{I}_{1} \boldsymbol{I}_{2}}, \underline{\boldsymbol{I}_{1}} \overline{\boldsymbol{I}_{2}}, \overline{\boldsymbol{I}_{1}} \underline{\boldsymbol{I}_{2}}, \overline{\boldsymbol{I}_{1} \boldsymbol{I}_{2}}\right)\right]
\end{aligned}
$$

Then, given the real function $f(x, y)=x \times x+y$, we may define its natural interval extension by $\boldsymbol{f}(\boldsymbol{x}, \boldsymbol{y})=\boldsymbol{x} \times \boldsymbol{x}+\boldsymbol{y}$, and we have that, e.g., $\boldsymbol{f}([2,3],[-1,5])=$ $[3,14]$.

Implementations of interval arithmetic use outward rounding to enlarge the domains computed so as not to violate the containment principle, should some bounds be unrepresentable with floating-point numbers [12].

Many numerical methods have been extended to use interval arithmetic $[22$, 24]. Given the system of nonlinear equations (1) and initial domains $\boldsymbol{I}_{\mathbf{1}}, \ldots, \boldsymbol{I}_{\boldsymbol{n}}$ for the variables, these methods are usually embedded into a branch-and-prune algorithm BaP (see Algorithm 1) that manages a set of boxes of domains to tighten. Starting from the initial box $\boldsymbol{D}=\boldsymbol{I}_{\mathbf{1}} \times \cdots \times \boldsymbol{I}_{\boldsymbol{n}}$, BaP applies a numerical method "prune" to tighten the domains in $\boldsymbol{D}$ around the solutions of System (1). It then bisects the resulting box along one of its dimensions whose width is larger than some specified threshold $\varepsilon$. The BaP algorithm eventually returns a set of boxes whose largest dimension has a width smaller than $\varepsilon$ and whose union contains all the solutions to Eq. (1). Note, however, that some boxes may eventually contain zero, one, or more than one solution.

Interval nonlinear Gauss-Seidel is a possible implementation for prune. It considers the $n$ unary projections:

$$
\begin{gathered}
f_{1}^{(1)}\left(x_{1}, I_{2}, \ldots, I_{n}\right)=0 \\
\ddots \\
f_{n}^{(n)}\left(I_{1}, \ldots, I_{n-1}, x_{n}\right)=0
\end{gathered}
$$

and uses any unidimensional root-finding method to tighten the domain of each variable $\boldsymbol{x}_{\boldsymbol{i}}$ in turn. Using a unidimensional Newton-Raphson root-finder leads 
to the Gauss-Seidel-Newton method [23], whose extension to intervals is the Herbort-Ratz method [11].

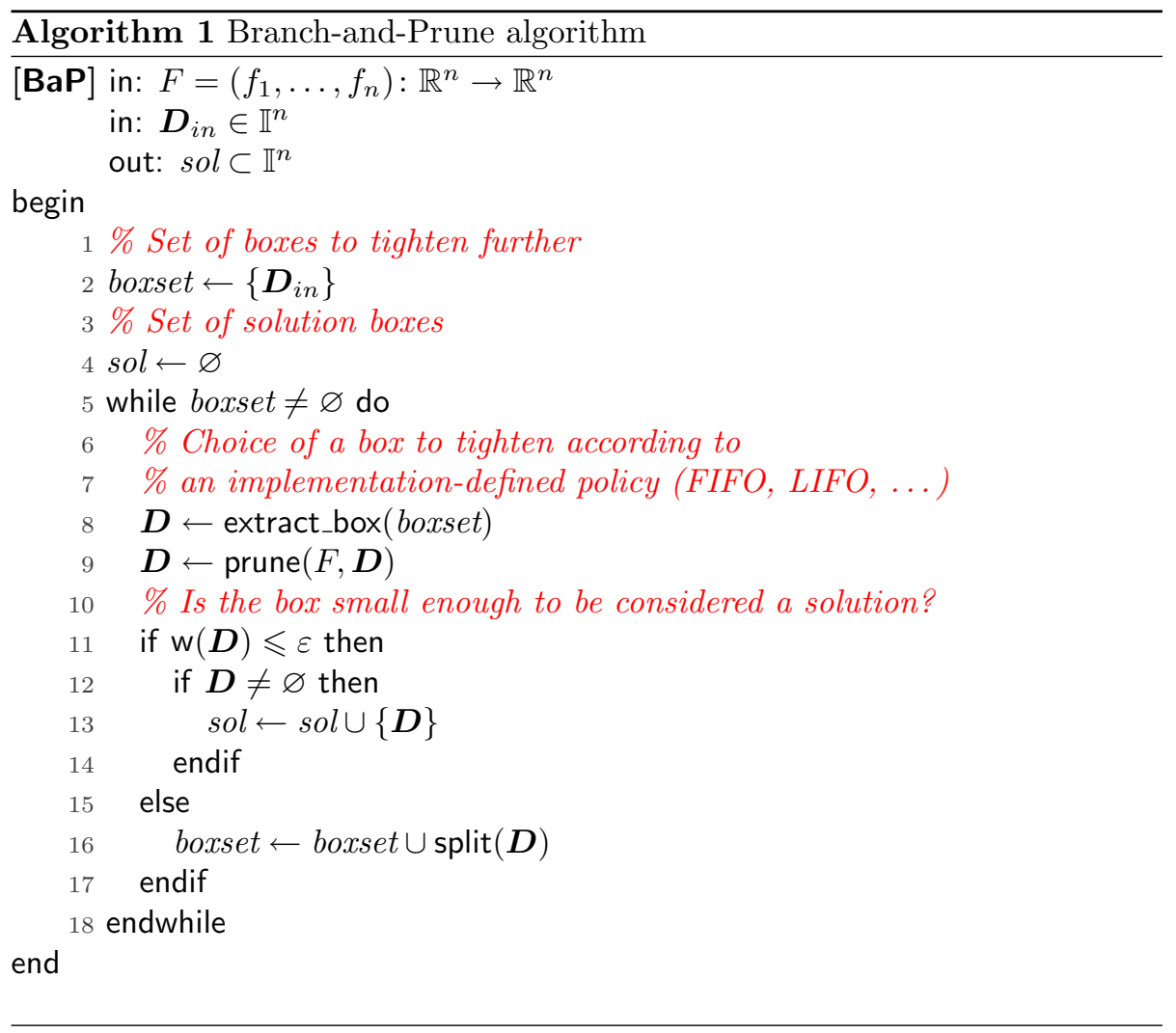

Let UN be the elementary step performed by one unidimensional Newton application to the projection $\boldsymbol{f}_{\boldsymbol{i}}^{(j)}$, where $\boldsymbol{i}$ and $\boldsymbol{j}$ may be different [23]. As soon as $\boldsymbol{D}$ is moderately large, it is very likely that each projection constraint will have many "solutions" that are not solutions of the original real system, and whose discarding slows down the computation. The Newton method will also fail to narrow down the domain of some $\boldsymbol{x}_{\boldsymbol{i}}$ if there is more than one solution to the corresponding projection constraint for the current box $\boldsymbol{D}$, thereby demanding more splitting in BaP. Achieving the right balance between the amount of work required by the prune method and the number of splitting performed overall is the key to maximum efficiency of BaP. In this very situation, experimental evidences show that trying harder to narrow down the domain of $\boldsymbol{x}_{\boldsymbol{i}}$ pays off [4]. A way to do it is to ensure that the canonical intervals $\left[\underline{\boldsymbol{I}_{\boldsymbol{j}}}, \underline{\boldsymbol{I}}_{\boldsymbol{j}}{ }^{+}\right]$and $\left[\overline{\boldsymbol{I}_{\boldsymbol{j}}}-\overline{\boldsymbol{I}_{\boldsymbol{j}}}\right]$, whose bounds are two consecutive floating-point-numbers, are solutions of $\boldsymbol{f}_{\boldsymbol{i}}^{(\boldsymbol{j})}\left(\boldsymbol{I}_{\mathbf{1}}, \ldots, \boldsymbol{I}_{\boldsymbol{j}-\mathbf{1}}, \boldsymbol{x}_{\boldsymbol{j}}, \boldsymbol{I}_{\boldsymbol{j}+\mathbf{1}}, \ldots, \boldsymbol{I}_{\boldsymbol{n}}\right)=0$. Algorithm bc3revise [4] ensures such a property (called box consistency of $\boldsymbol{x}_{\boldsymbol{j}}$ w.r.t. the constraint $\boldsymbol{f}_{\boldsymbol{i}}=0$ and $\boldsymbol{D})$ for a projection $\boldsymbol{f}_{i}^{(j)}$. A simple method to implement it combines 
a dichotomic process with Newton-Raphson steps to isolate the leftmost and rightmost solutions included in $\boldsymbol{D}$ of each projection constraint.

Example 2 Consider the constraint $f(x)=(x-1.5)(x-2)(x-3)=0$ and the domain $\boldsymbol{I}=[1,4]$ for $x$ (See Fig. 1). The UN method leaves $\boldsymbol{I}$ unchanged because the derivative of $f$ over the initial domain contains 0 while bc3revise narrows down $\boldsymbol{I}$ to $\boldsymbol{I}^{\prime}=[1.5,3]$, which is the smallest interval included in $\boldsymbol{I}$ that contains all the solutions to the interval constraint $\boldsymbol{f}(\boldsymbol{x})=0$.

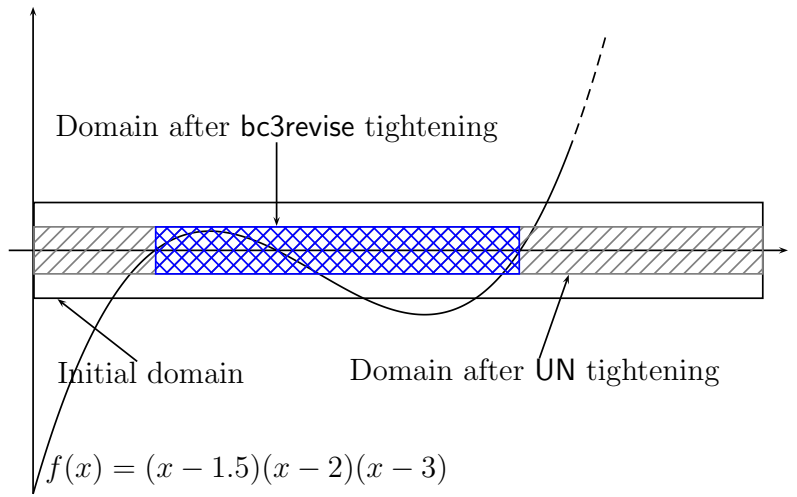

Figure 1: Comparison of UN and bc3revise

Interval constraint methods [3] combine interval arithmetic - to reliably solve a system of real equations without loss of solutions - and smart propagation algorithms [19], to take advantage of its possible sparsity (some variables may not occur in all constraints).

Algorithm bc3 [4] (see Algorithm 2) is such a method, which relies on the pruning operator bc3revise to tighten domains. It is akin to a free-steering generalized nonlinear Gauss-Seidel method with a twist [7]: as shown in Algorithm 2, the set of projections on which bc3revise is applied contains all the possible projections from the equation system, and not $n$ of them only.

Algorithm bc3, or one of its variations, is often used as a basis to reliably solve nonlinear constraint systems, though its use of the at most $n^{2}$ projections of a system of $n$ equations on $n$ variables makes it a bad choice for large dense problems due to the overwhelming number of projections it then has to consider. It is also sensitive to a problem that plagues other interval constraint algorithms, whereby many calls of the contracting operators lead to no reduction of the domains at all. Figure 2 shows this situation for bc3 on twelve standard test problems to be presented in Section 4: calls to bc3revise are separated into three categories (very effective calls leading to a reduction of domain size by more than $10 \%$, effective calls leading to a reduction of domain size by less than $10 \%$, and useless calls leading to no reduction at all). As we can see, the majority of the work performed is essentially useless for almost all problems. 

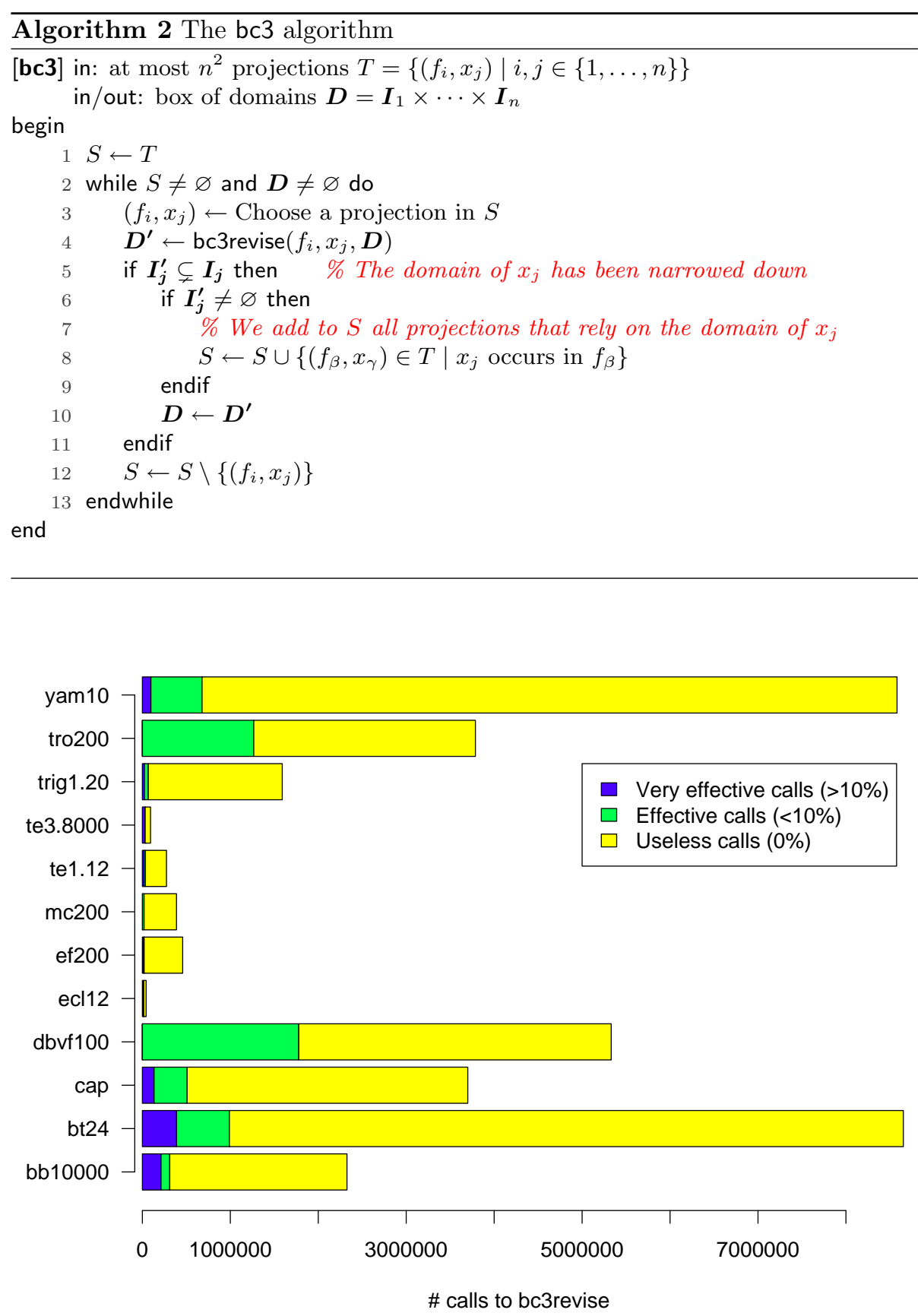

Figure 2: Effectiveness of bc3 
Since no fail-safe efficient strategy exists for choosing the right contracting operator (line 3 of Algorithm 2) at the right time, the standard implementation uses a queue to represent $S$ (the contracting operators are applied in the order they are inserted).

These inefficiencies may have two different non-exclusive causes: either some of the at most $n^{2}$ projections never lead to any reduction, and therefore only clutter the propagation queue; or the effectiveness of projections varies widely during the solving process and may oscillate from nothing to good.

In the first case, optimizing bc3 boils down to statically identifying the best projections and using only these ones; in the second case, we have to keep all $n^{2}$ projections and find a means to consider at any time during the solving process only those projections with good tightening potential.
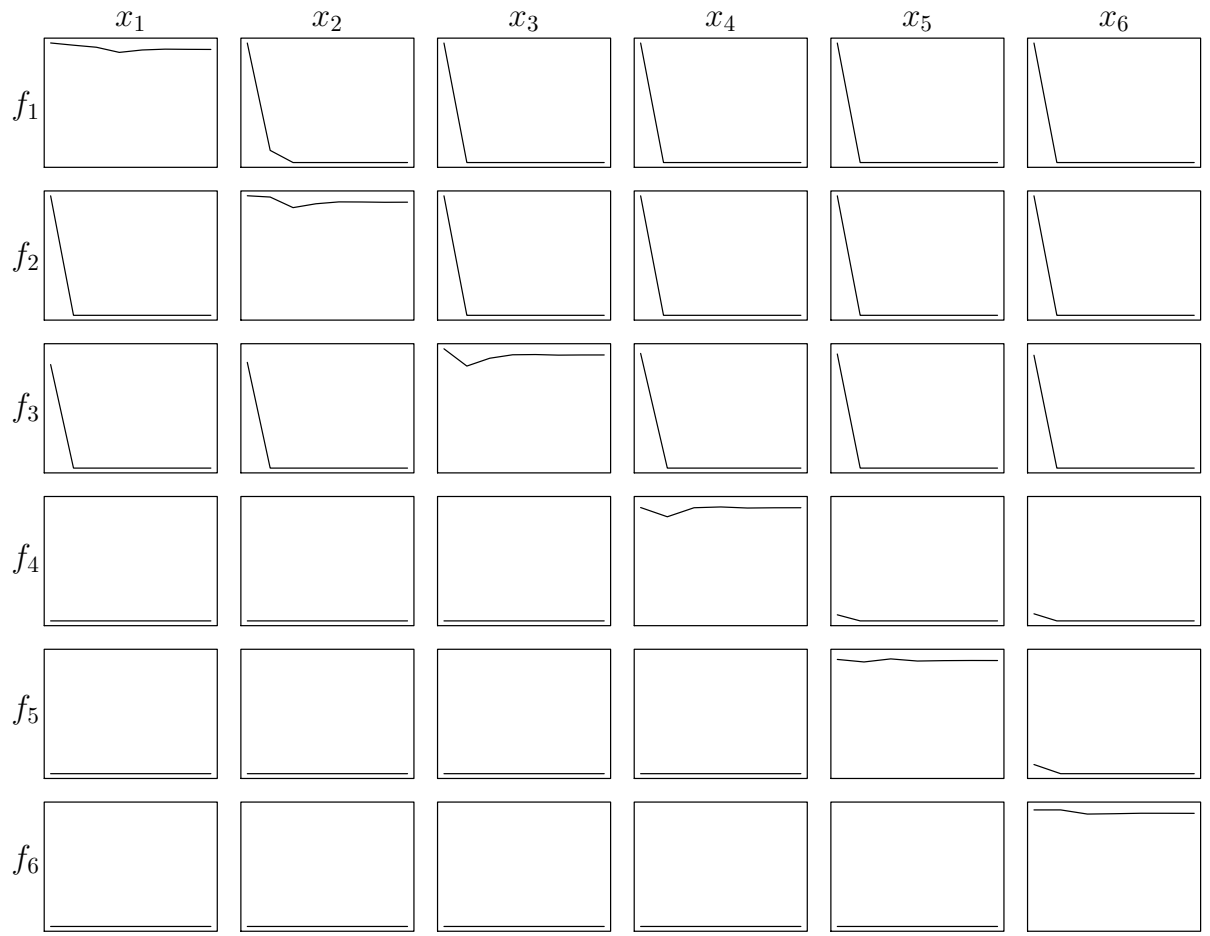

Figure 3: History of narrowing effectiveness per projection for Moré-Cosnard-6; in ordinate, the efficiency in domain reduction percentage; in abscissa, calls to the corresponding projection.

As the following examples show, it appears that, depending on the problem considered, both situations may arise. Consider the Moré-Cosnard problem [21] of dimension 6: Figure 3 shows the history of effectiveness (in ordinate, percentage of reduction obtained in the range $[0,1]$ ) of each projection when solving 
it with bc3 (abscissa goes from the first use of $\left(f_{i}, x_{j}\right)$ to its last use $\left.{ }^{2}\right)$. One may easily see that the only useful projections are the ones on the diagonal. As a side note, we may also remark that some projection (e.g., $\left(f_{1}, x_{2}\right),\left(f_{2}, x_{3}\right)$, ... ) perform well the first time they are used, and then consistently badly afterwards. This does not bode well for identifying statically which are the best projections to retain.

On the other hand, consider the sparse problem Trigexp1 [18] for $n=6$ : The history of effectiveness given in Figure 4 shows that there are more than $n$ useful projections (for example, $\left(f_{5}, x_{4}\right)$ and $\left(f_{4}, x_{3}\right)$ should probably both be retained). Furthermore the effectiveness of each projection varies widely during the solving process, and some projections that are not very good in the beginning become good or average afterwards (e.g., $\left.\left(f_{5}, x_{4}\right)\right)$, while some projections that are very good in the beginning become quite bad after some time (e.g., $\left(f_{2}, x_{2}\right)$ ).
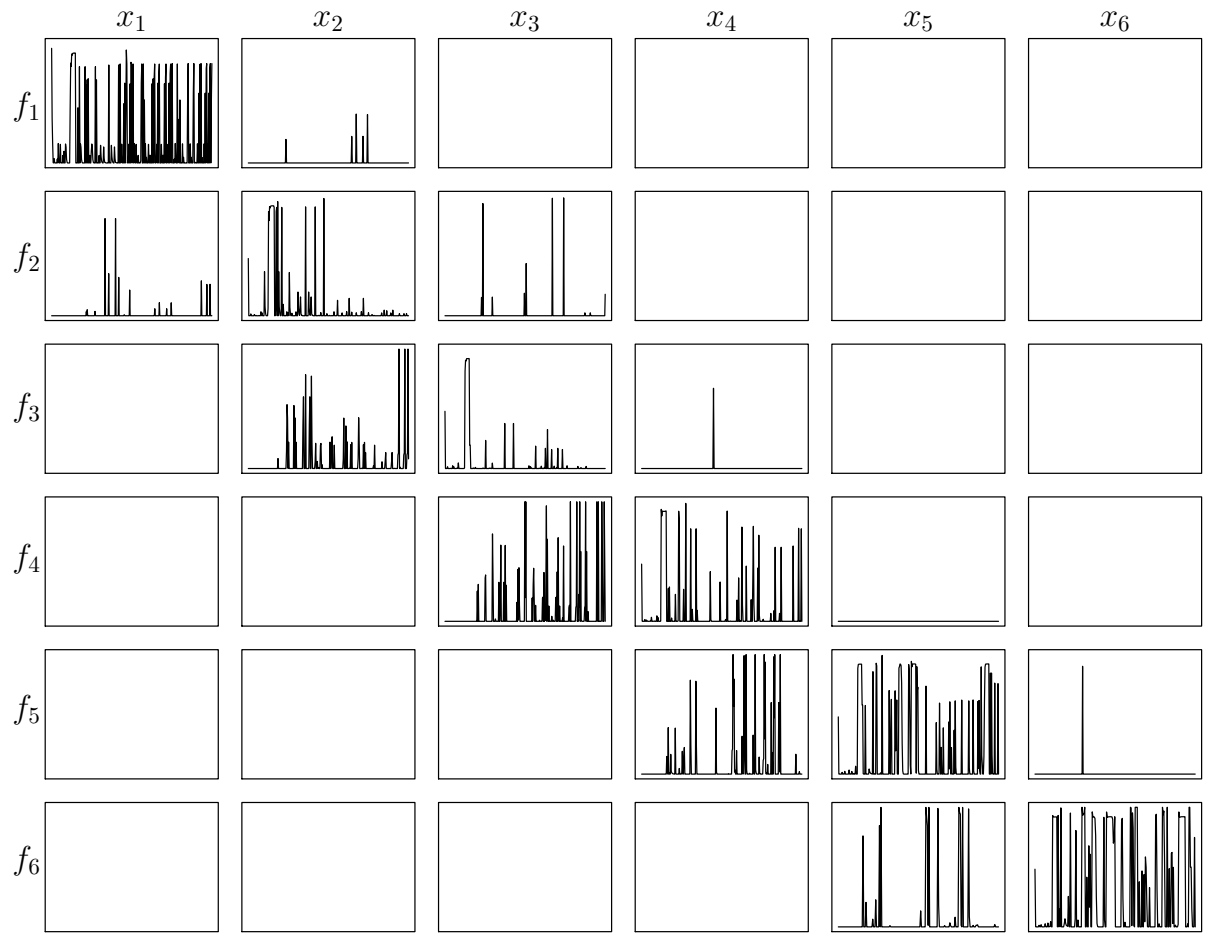

Figure 4: History of narrowing effectiveness per projection for Trigexp1 $(n=6)$

These examples should convince us that we have to keep all projections for consideration in bc3, and that we must resort to some dynamic selection

\footnotetext{
${ }^{2}$ Note that, for Moré-Cosnard as well as for the next example Trigexp1, each projection is used almost as many times as the others in an implementation of bc3 in which $S$ is managed as a queue.
} 
scheme to apply bc3revise only on those projections that offer the best narrowing potential at some point in the solving process.

\section{Speeding-up solving through Reinforcement Learning}

Reinforcement learning [26] is a sub-area of machine learning considering unsupervised agents that iteratively refine their strategy for choosing actions in an uncertain environment so as to maximize a long-term reward. Agents refine their knowledge of the environment by observing the effect of the most recently chosen actions. Hence, they have to achieve the optimal trade-off between exploration - testing the different actions at hand - and exploitationperforming the actions that have the greatest potential reward. The problem is compounded in an ever-evolving environment, that is when the probability of a reward for an action may vary.

A standard problem considered in reinforcement learning is the multi-armed bandit problem [26]: given $k$ slot machines with payoff probabilities unknown to the player and some time horizon, find the sequence of levers to pull in order to maximize the gains. In this problem, the action chosen is represented by the number associated with the lever to pull, the reward is the gain obtained by pulling the lever chosen, and the long-term objective is to maximize the cumulated rewards at the time horizon. The non stationary variant of the problem involves slot machines with varying payoff probabilities [1,2].

A close look at our problem allows us to draw an analogy between the selection process of projections in bc3 and the non stationary multi-armed bandit problem: in our context, the $k$ levers are the at most $n^{2}$ projections, and their payoff is the relative domain reduction ${ }^{3}$ their use in bc3revise leads to. We use the relative domain reduction instead of the absolute one as a measure of efficiency in order not to favor too much the projections used early when the domains of variables are large to the detriment of projections applied on smaller domains.

No time horizon is given in bc3. However, by maximizing the sum of relative domain reductions, we expect both to avoid applying bc3revise on projections that do not lead to any reduction, and to reduce the overall number of calls to bc3revise, thereby accelerating the computation of solutions ${ }^{4}$.

\subsection{Adaptation of reinforcement learning to bc3}

A difficulty of the adaptation of the reinforcement learning approach to our problem of selecting the best projections is that, especially in big or dense prob-

\footnotetext{
${ }^{3}$ The relative reduction is defined by $\left(\mathrm{w}\left(\boldsymbol{I}_{\boldsymbol{j}}^{\boldsymbol{b}}\right)-\mathrm{w}\left(\boldsymbol{I}_{\boldsymbol{j}}^{\boldsymbol{a}}\right)\right) / \mathrm{w}\left(\boldsymbol{I}_{\boldsymbol{j}}^{\boldsymbol{b}}\right)$ where $\mathrm{w}\left(\boldsymbol{I}_{\boldsymbol{j}}^{\boldsymbol{b}}\right)\left(\right.$ resp. $\left.\mathrm{w}\left(\boldsymbol{I}_{\boldsymbol{j}}^{\boldsymbol{a}}\right)\right)$ is the width of the domain of $x_{j}$ before (resp. after) applying bc3revise on $\left(f_{i}, x_{j}\right)$.

${ }^{4}$ Once again, in interval constraint programming, a solution is a Cartesian product of domains whose largest width is smaller than a predefined threshold, and for which it is not possible to prove that it does not contain any point satisfying the system.
} 
lems, the number of projections among which to choose is so large that a lot of time can be spent exploring alternatives. Consequently, we have retained the recency-weighted average (rwa) [26, Chap. 2, Sect. 6] as a reasonable reinforcement learning method for our purpose, it being more exploitation-oriented than most other methods.

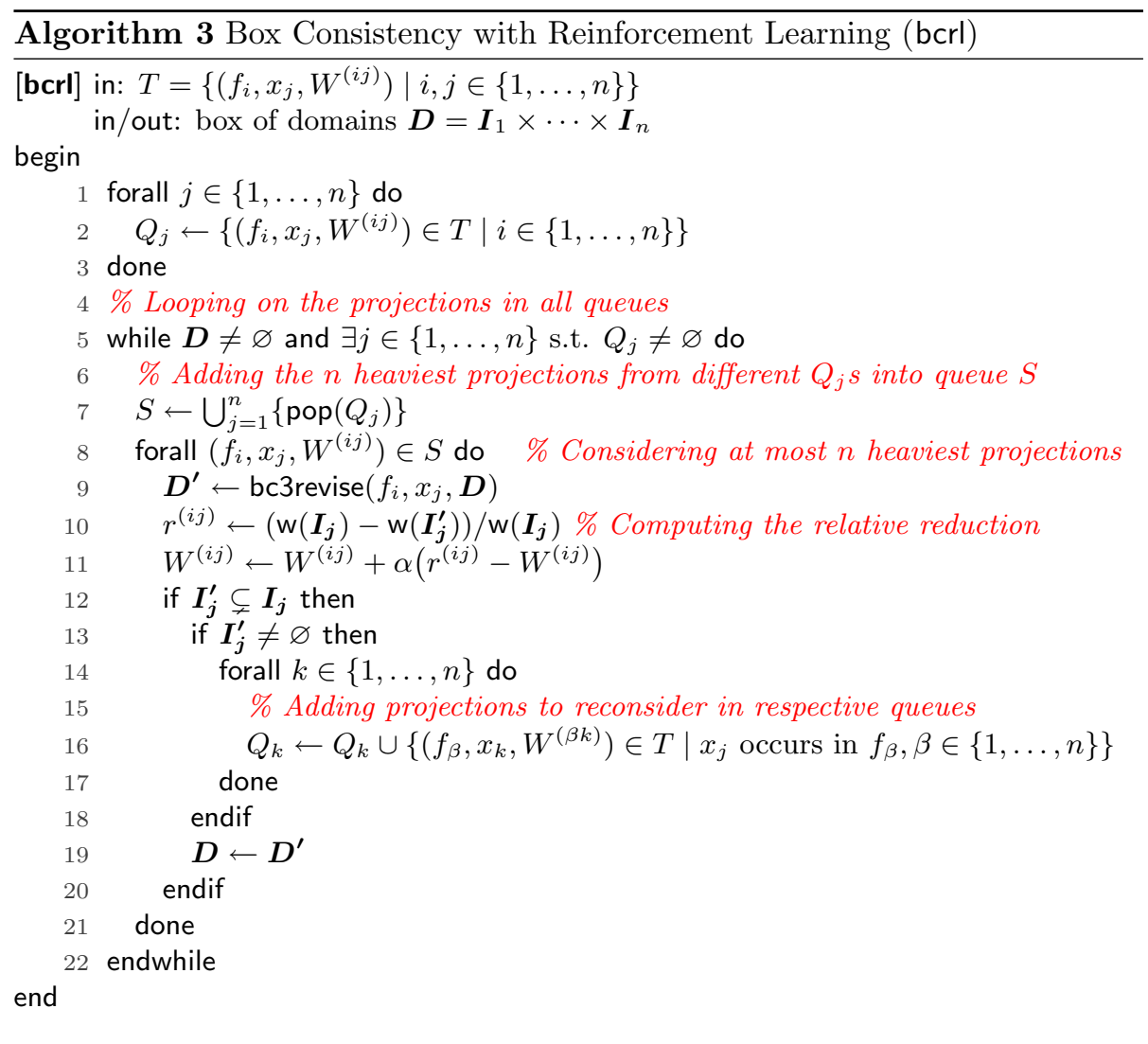

Algorithm rwa is a standard method to solve non stationary reinforcement learning problems. It associates to each possible action a weight that measures its interest. This weight is a weighted average of all past rewards, hence the name. At each iteration, the action with the highest weight is chosen, its reward observed, and its weight updated accordingly. This method adopts a pure exploitation strategy since alternative choices are never explored.

In our context, using rwa means associating a weight $W^{(i j)}$ with each projection $\left(f_{i}, x_{j}\right)$; the set $S$ in bc3 (see Algorithm 2) is replaced by a priority queue (heaviest weights available first). Line 3 is then replaced by the extraction of the projection with heaviest weight. Let $r^{(i j)}$ be the relative reduction obtained on Line 4 . The weight $W_{k+1}^{(i j)}$ that takes into account the $k$ past payoffs and the 
most recent one $r_{k+1}^{(i j)}$ is obtained with the formula:

$$
W_{k+1}^{(i j)}=W_{k}^{(i j)}+\alpha\left(r_{k+1}^{(i j)}-W_{k}^{(i j)}\right)
$$

where $\alpha$ is a constant parameter between 0 and 1 that monitors the importance granted to the past payoffs w.r.t. the current one.

Using one priority queue $S$ for all the projections, as done in bc3, is a suboptimal strategy here in that it may create propagation cycles leading to overall slow convergence phenomena [17]. As an illustration, consider two projections $p_{1}$ and $p_{2}$ such that when $p_{1}$ is applied, $p_{2}$ is inserted in $S$ and conversely. It could well be the case that $p_{1}$ and $p_{2}$ are applied cyclically with enough success so that the other projections are not considered. Such a phenomenon is in general counterproductive in the long term: even though $p_{1}$ and $p_{2}$ produce good relative reductions, they do not reduce the domains of all the variables and do not consider all the constraints.

To avoid this, we use one priority queue $Q_{j}$ per variable $x_{j}$; when it needs to be reconsidered, the projection $\left(f_{i}, x_{j}\right)$ is always pushed in $Q_{j}$. The resulting algorithm bcrl is presented in Algorithm 3. Algorithm bcrl contains an inner loop over at most $n$ projections on $n$ different variables (less than $n$ projections if some queues are temporarily empty) in addition to the while loop to reach a fixed-point, which was already present in bc3.

Note: In order to rigorously validate our results and to assess the impact of the various choices we made, we have tested using one propagation queue per variable with bc3 as well. For all the problems considered here, the computational time required is essentially similar to the one required by bc3 with one propagation queue only (see Figure 5).

\subsection{Setting up bcrl}

In order to obtain a fully defined algorithm for bcrl, we need to set two interdependent parameters: the value of $\alpha$ and the value of the initial weights.

According to Eq. (4), $W^{(i j)}$ is a weighted average of the past payoffs and of the initial weight $W_{0}^{(i j)}$ :

$$
W_{k}^{(i j)}=(1-\alpha)^{k} W_{0}^{(i j)}+\sum_{l=1}^{k} \alpha(1-\alpha)^{(k-l)} r_{l}^{(i j)}
$$

Consequently, for a small $\alpha$ (e.g., $\alpha=0.1)$, the weight $\alpha(1-\alpha)^{(k-l)}$ of the payoffs will decrease only slightly with their age, with the exception of the initial "payoff", whose weight remains important. By contrast, with a large $\alpha$ (e.g., $\alpha=0.9$ ), the weights of the payoffs decrease fast with their age, with the most recent payoff being much favored.

With a large $\alpha$, a projection may see its weight plunge the first time it performs badly, while the aftermath of such an event would be dampened with 


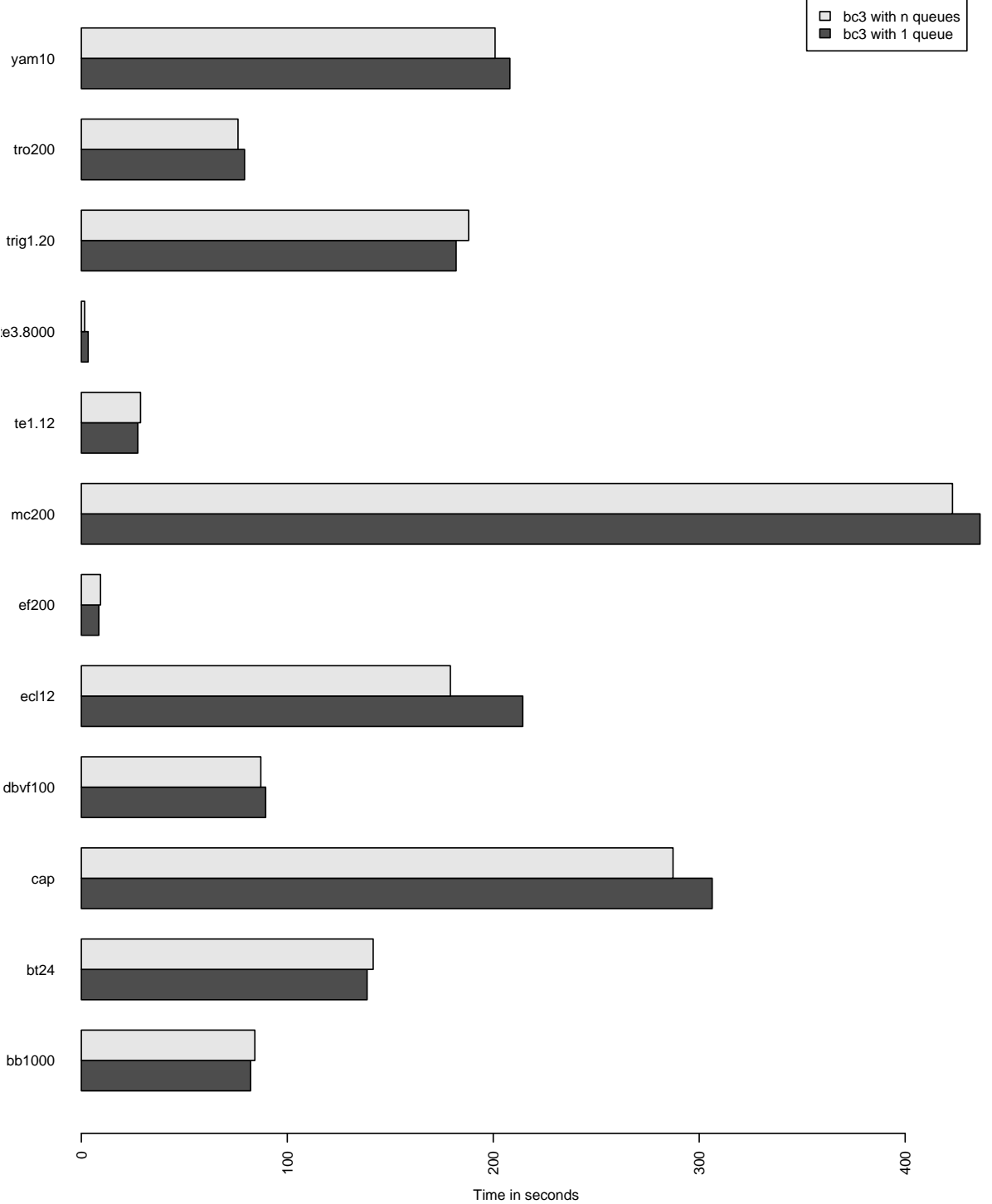

Figure 5: Comparing bc3 with only one queue and with one queue per variable 
Table 1: Incidence of $\alpha$ on computation times for bcrl-1

\begin{tabular}{lrrrrr}
\hline Problem / $\alpha$ & 0.1 & 0.3 & 0.5 & 0.7 & 0.9 \\
\hline \hline bb10000 & 93 & 68 & 63 & 56 & $\mathbf{4 9}$ \\
bt24 & 132 & 130 & 152 & 139 & $\mathbf{1 1 7}$ \\
cap & 132 & 124 & 125 & 117 & $\mathbf{1 1 5}$ \\
dbvf100 & $\mathbf{4 4}$ & $\mathbf{4 4}$ & $\mathbf{4 4}$ & $\mathbf{4 4}$ & $\mathbf{4 4}$ \\
ecl12 & 291 & 285 & 284 & $\mathbf{2 8 1}$ & 283 \\
ef200 & $\mathbf{8}$ & $\mathbf{8}$ & $\mathbf{8}$ & $\mathbf{8}$ & 9 \\
mc200 & $\mathbf{6 7 4}$ & 749 & 920 & 1195 & 1331 \\
te1.12 & $\mathbf{2 5}$ & 26 & 26 & 28 & 26 \\
te3.8000 & $\mathbf{1}$ & $\mathbf{1}$ & $\mathbf{1}$ & $\mathbf{1}$ & $\mathbf{1}$ \\
trig1.20 & $\mathbf{8 2}$ & $\mathbf{8 2}$ & 83 & 85 & $\mathbf{8 2}$ \\
tro200 & $\mathbf{4 5}$ & 46 & $\mathbf{4 5}$ & $\mathbf{4 5}$ & $\mathbf{4 5}$ \\
yam10 & $\mathbf{4 6}$ & 47 & 47 & 47 & 47 \\
\hline
\end{tabular}

Times in seconds on an Intel Pentium IV at $3.8 \mathrm{GHz}$ (rounded to the nearest sec.)

Boldfaced time: best time for a benchmark

a small $\alpha$ by the cumulative effect of its past history. On the other hand, the use of a small $\alpha$ requires extra care when initializing the weights $W_{0}$. In any case, a consequence of the weight update formula (5) is that the initial weight $W_{0}$ may be an important bias of the rwa method.

Without further information, we first decide to initialize all weights to 1 , giving equal importance to all projections. Table 1 shows the impact of $\alpha$ on computation time for these initial weights (Algorithm bcrl-1). Both 0.1 and 0.9 seem good contenders for the choice as default values.

Figure 6 presents a comparison of the number of effective and useless calls for bc3 and bcrl-1 for standard test problems to be described in Section 4 . For all problems, the lowest bar corresponds to bc3 while the topmosts correspond, from bottom to top, to bcrl-1 for $\alpha=0.1$ and $\alpha=0.9$.

Overall, bcrl-1 reduces the total number of calls to bc3revise (and therefore, the solving time - see Figure 8, page 18) for most problems. On closer look, it appears that bcrl-1 requires more calls than bc3 on problem mc200, though the number of effective calls is also increased. For this dense problem, the number of projections is large $\left(200^{2}\right)$, and bcrl- 1 requires a long time to discover that it possesses a static transversal (see Page 8) because all projections have the same weight initially, and are then all considered in turn at least once at the beginning. This effect is worsened with $\alpha=0.9$ by a perverse side-effect of it selecting effective projections more often than with $\alpha=0.1$ : out of 602199 calls to bc3revise, $122002(20.25 \%$ ) lead to some insubstantial reduction (less than $10 \%$ ); by contrast, using $\alpha=0.1$ yields 571999 calls to bc3revise (only $5 \%$ less than with $\alpha=0.9$ ), out of which only $24033(4 \%)$ lead to some reduction less than $10 \%$. Each successful call to bc3revise leads to testing whether to include in the propagation queues the projections that depend on the variable reduced 


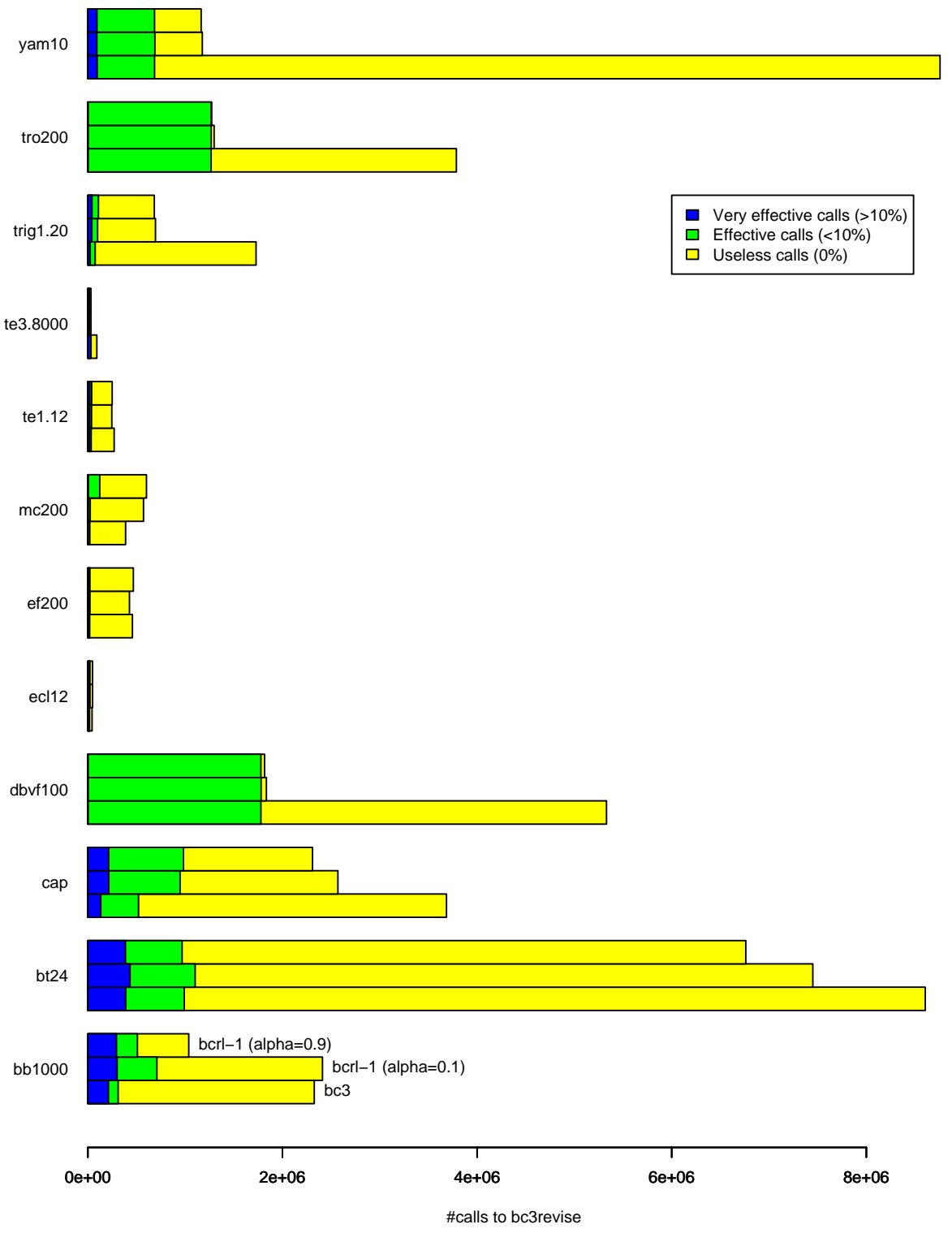

Figure 6: Effectiveness of bcrl-1 ( $\alpha=0.1$ and $\alpha=0.9)$ vs. bc3 
Table 2: Incidence of $\alpha$ on computation times for bcrl-j

\begin{tabular}{lrrrrr}
\hline Problem / $\alpha$ & 0.1 & 0.3 & 0.5 & 0.7 & 0.9 \\
\hline \hline bb10000 & $\mathbf{3 4}$ & $\mathbf{3 4}$ & $\mathbf{3 4}$ & 36 & 47 \\
bt24 & 155 & 147 & 158 & 144 & $\mathbf{1 2 1}$ \\
cap & 131 & 126 & 119 & 116 & $\mathbf{1 1 5}$ \\
dbvf100 & 44 & 44 & $\mathbf{4 3}$ & $\mathbf{4 3}$ & 44 \\
ecl12 & 278 & 270 & 275 & 269 & $\mathbf{2 6 8}$ \\
ef200 & $\mathbf{8}$ & $\mathbf{8}$ & $\mathbf{8}$ & $\mathbf{8}$ & 9 \\
mc200 & $\mathbf{3 9}$ & $\mathbf{3 9}$ & $\mathbf{3 9}$ & $\mathbf{3 9}$ & $\mathbf{3 9}$ \\
te1.12 & $\mathbf{2 5}$ & $\mathbf{2 5}$ & 26 & 26 & 26 \\
te3.8000 & 6 & $\mathbf{5}$ & $\mathbf{5}$ & $\mathbf{5}$ & $\mathbf{5}$ \\
trig1.20 & 88 & $\mathbf{8 5}$ & 86 & 87 & 86 \\
tro200 & $\mathbf{4 5}$ & $\mathbf{4 5}$ & $\mathbf{4 5}$ & $\mathbf{4 5}$ & $\mathbf{4 5}$ \\
yam10 & $\mathbf{4 6}$ & $\mathbf{4 6}$ & $\mathbf{4 6}$ & $\mathbf{4 6}$ & 47 \\
\hline
\end{tabular}

Times in seconds on an Intel Pentium IV at $3.8 \mathrm{GHz}$ (rounded to the nearest sec.)

Boldfaced time: best time for a benchmark

(see Line 16 in Algorithm bcrl, Page 10). For a large dense problem such as mc200, this process takes a lot of time because there are $200^{2}-1$ projections to consider each time. A solution to this problem is to introduce a so-called improvement factor $\gamma$ and to forbid propagation (that is, to bypass Line 17 in bcrl) whenever the reduction achieved by a call to bc3revise is smaller than $\gamma \%$. When using an improvement factor of $10 \%$, bcrl-1 with $\alpha=0.9$ becomes twice as fast as with $\alpha=0.1$ on mc200. In addition, setting mc200 aside, the choice of $\alpha=0.9$ leads to better performances overall than $\alpha=0.1$.

As a consequence, we decide to favor the reactivity offered by $\alpha=0.9$, and we choose it as the default value in the rest of this paper.

\subsection{Enhancing bcrl with an initial guess}

In order to achieve good performances even for large dense problems with static transversals, we have to use some information at the beginning of the solving process to preset the weights to favor some projections over others. If our initialization heuristic is good, we expect that the best projections will be used more often than the others from the very start.

The heuristic chosen works as follows: we compute the interval Jacobian $\boldsymbol{J}$ of the system for the initial box and set $W^{(i j)}$ to the sum of the mignitude ${ }^{5}$ of $\boldsymbol{J}_{i j}$ normalized to the range $[0,0.5]$ and of the magnitude ${ }^{6}$ of $\boldsymbol{J}_{i j}$ normalized to the range $[0,0.5]$. The weight thus lies in the range $[0,1]$.

Intuitively, the interval Jacobian indicates the steepness of the projections, i.e. which projections are more likely to allow reducing the domain of their

\footnotetext{
${ }^{5} \operatorname{mig} \boldsymbol{I}=\min \{|a| \mid a \in \boldsymbol{I}\}$

${ }^{6} \operatorname{mag} \boldsymbol{I}=\max \{|a| \mid a \in \boldsymbol{I}\}$
} 


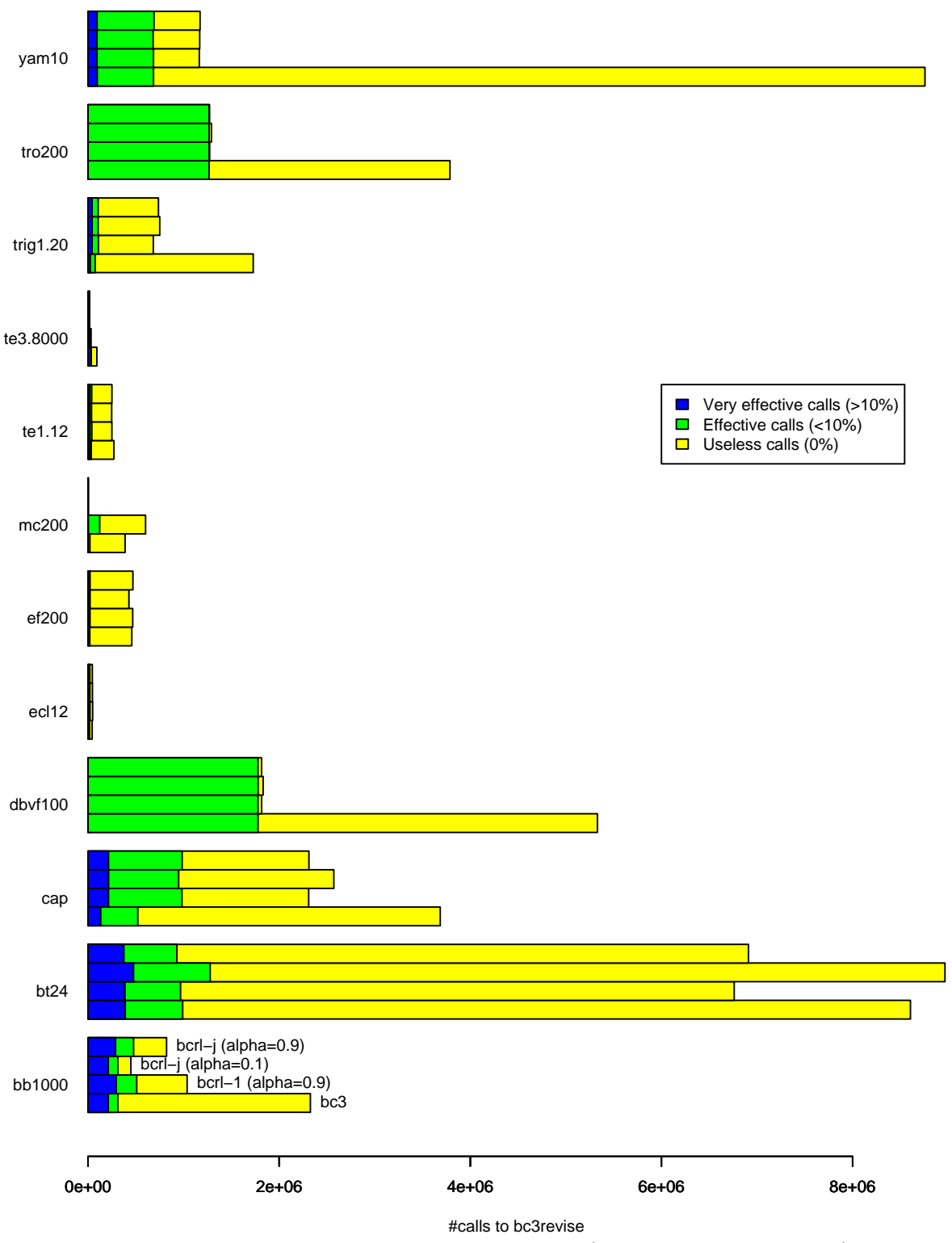

Figure 7: Effectiveness of bc3 vs. bcrl-j ( $\alpha=0.1$ and $\alpha=0.9)$ 
associated variable. The mignitude of an entry in the Jacobian gives a worst-case information on the steepness while the magnitude gives a best-case information. The heuristic chosen mixes both equally.

In the following, times reported always take into account the time spent in computing the initial weights.

The resulting instance of bcrl is called bcrl-j. Table 2 presents the incidence of $\alpha$ for bcrl-j. Note how bcrl-j seems less sensitive to the value of the parameter $\alpha$ than bcrl-1. Indeed, since rwa follows a pure exploitation strategy, enhancing it with a good initial guess yields a more focused exploitation. Since the heuristic is good on our test set, the influence of $\alpha$ is dampened. However, we think it is a better choice to keep $\alpha=0.9$ as the default value for bcrl-j in case the heuristic would fail on some problem, or when the problem does not have a clear transversal.

Figure 7 graphically compares the number of calls to bc3revise required to solve our set of test problems. The lowest bar corresponds to bc3, the middle bar to bcrl-1, and the topmost bars to bcrl-j. As expected, bcrl-j performs consistently better than bc3 and bcrl-1. For Problem mc200, it achieves such a speed-up that it is not even visible on the chart. The reason is that the heuristic clearly identifies the transversal in this problem and initializes the weights accordingly, allowing bcrl-j to purely exploit this transversal. For problems without a clear transversal (e.g. ef200), the heuristic does not hinder proper learning.

\section{Evaluating bcrl}

In order to assess the quality of our new algorithms, we have selected a set of twelve standard nonlinear problems [14] of various sizes, various characteristics (quadratic constraints, polynomial constraints, non-polynomial constraints involving sines, cosines, logarithms and exponentials), and various sparsities (dense problems, in which all variables occur in all equations, and sparse problems, with only a small subset of the variables in each equation). The characteristics for all these problems are synthesized in Table 3. The Size column indicates the number $n$ of variables and equations (all the problems are square); the Sparsity column gives the sparsity index defined as the number $p$ of possible projections divided by $n^{2}$ ( $p$ is equal to $n^{2}$ for dense problems such as mc200 but may be much smaller for sparse problems). The initial domains for all test problems are those given on the COPRIN web page [14].

Figure 8 presents the computation times for the three algorithms considered. All the experiments were conducted on an Intel Pentium IV at $3.8 \mathrm{GHz}$ with 2GB of RAM and a Standard Unit Time equal to $50.4 \mathrm{~s}$ (this is defined as the time required for performing $10^{8}$ evaluations of the function Shekel 5 [5]). The constraint solving environment used is a $\mathrm{C}++$ library written from scratch by the authors. The times reported correspond to the enclosing of all the solutions in boxes of domains whose largest dimension is smaller than $10^{-8}$.

As noted previously, bc3 performs poorly on dense problems (e.g., mc200) 


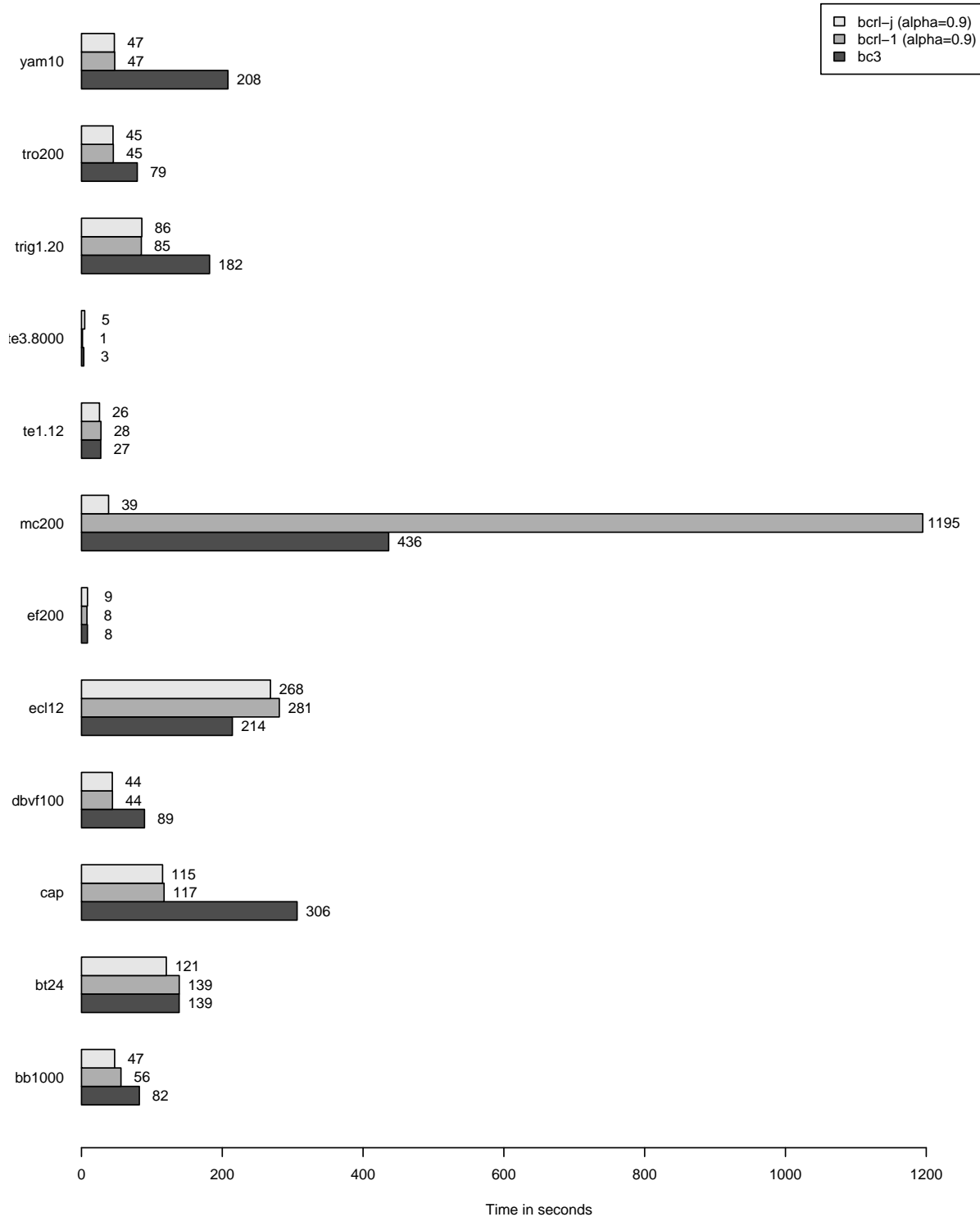

Figure 8: Solving test problems with and without learning (times rounded to the nearest second) 
Table 3: Test problems

\begin{tabular}{llrrc}
\hline Name & Code & Size & Sparsity & Constraints \\
\hline \hline Broyden-banded & bb10000 & 10000 & $0.07 \%$ & quadratic \\
Broyden tridiagonal & bt24 & 24 & $10.95 \%$ & quadratic \\
Caprasse & cap & 4 & $100.00 \%$ & polynomial \\
Discrete Boundary Value Fun. & dbvf100 & 100 & $2.93 \%$ & polynomial \\
Extended Crag-Levy & ecl12 & 12 & $14.58 \%$ & non-polynomial \\
Extended Freudenstein & ef200 & 200 & $1.00 \%$ & polynomial \\
Moré-Cosnard & mc200 & 200 & $100.00 \%$ & polynomial \\
Trigexp 1 & te1.12 & 12 & $23.61 \%$ & non-polynomial \\
Trigexp 3 & te3.8000 & 8000 & $0.04 \%$ & non-polynomial \\
Trigo1 & trig1.20 & 20 & $100.00 \%$ & non-polynomial \\
Troesch & tro200 & 200 & $1.49 \%$ & non-polynomial \\
Yamamura & yam10 & 10 & $100.00 \%$ & polynomial \\
\hline
\end{tabular}

due to the sheer number of projections to consider. The results for bcrl-1 are consistent with the observation made on Figure 6: the method is often better than bc3 though it can perform poorly on dense problems with a clear static transversal (e.g., mc200), spending too much time in exploration. Though not always the fastest method, bcrl-j appears the most regular in its results since it always solves the problems in a time that is close to that of the best method.

\section{$5 \quad$ Related works}

As said previously, there are already well established results for optimizing the resolution of systems of linear real equations with first-order methods that require strict diagonal dominance of the coefficient matrix $[23,6]$.

The literature dealing with nonlinear systems often revolves around methods that linearize them in order to exploit the results on linear systems: many papers consider an interval Newton-Gauss-Seidel method (aka Hansen-Sengupta's method [10]) that solves the linear system obtained from the local first-order expansion of nonlinear terms obtained with Newton's method by a preconditioned Gauss-Seidel method [15]. Accordingly, a lot of work is then devoted to finding the best preconditioners [16].

Sotiropoulos (sot) et al. [25] have an original approach in this respect: they select a transversal for a polynomial system at the beginning of the computation by looking at the syntactic structure of the equations (variables with the largest degree in the system, for example), and by using numerical considerations only to break ties. In their paper, the static transversal thus obtained is then used in an interval Newton-Gauss-Seidel algorithm.

Another approach uses a Gauss-Seidel-Newton method as presented in the introduction: Herbort and Ratz [11] (hr) compute the Jacobian $\boldsymbol{J}$ of the equation system w.r.t. the initial box $\boldsymbol{D}$, and they select projections according to whether 
Table 4: Comparing related work with solving by learning

\begin{tabular}{|c|c|c|c|c|}
\hline Problem & $\mathrm{hr}$ & gh & sot & $\begin{array}{c}\text { bcrl-j } \\
(\alpha=0.9)\end{array}$ \\
\hline bb10000 & 1760 & $\overline{\mathrm{TO}}$ & $\overline{\mathrm{TO}}$ & 47 \\
\hline bt24 & 228 & TO & 198 & 121 \\
\hline cap & TO & 237 & 112 & 115 \\
\hline dbvf100 & 41 & TO & 35 & 44 \\
\hline ecl12 & 2266 & 677 & NA & 268 \\
\hline ef200 & TO & 329 & TO & 9 \\
\hline mc200 & 3 & 860 & TO & 39 \\
\hline te1.12 & TO & 155 & NA & 26 \\
\hline te3.8000 & 1 & 71 & NA & 5 \\
\hline trig1.20 & 116 & 303 & NA & 86 \\
\hline tro200 & 48 & TO & NA & 45 \\
\hline yam10 & 48 & 3133 & 38 & 47 \\
\hline \multicolumn{5}{|c|}{$\begin{array}{l}\text { Times in seconds on an Intel Pentium IV at } 3.8 \mathrm{GHz} \text { (rounded to the nearest sec.) } \\
\text { TO: Time out }(7200 \mathrm{~s}) \text { reached }\end{array}$} \\
\hline
\end{tabular}

the corresponding entry in the Jacobian straddles zero or not. Their method is not completely static since they recompute the Jacobian after each outer step of Gauss-Seidel. In addition, it theoretically allows for the choice of more than $n$ projections.

In settings more similar to ours, Granvilliers and Hains [9] (gh) try to optimize bc3 by applying bc3revise on all projections at the beginning, and by selecting only the ones that tightened variables' domains the most. The choice of the projections retained is reconsidered whenever some splitting occurs in Algorithm BaP. For problems that lead to a lot of splitting, the method becomes computationally expensive.

Table 4 compares these three methods with bcrl-j. Note that these results should be taken with great care since we used our own implementation of these methods in the same environment as for bcrl-j, and that our only sources of information are the papers in which they were originally presented. With that proviso in mind, we see that bcrl-j outperforms hr and gh most of the times. With the exception of problem $b b 10000$ where it takes more than 150 times longer than bcrl-j, the method sot is quite good on the benchmarks it can handle, that is those with strictly polynomial expressions, which should lead us to investigate how to harness the power of its heuristics in our settings. Performance of $\mathrm{hr}$ is stunning on mc200. We suspect that it is because this problem has only one solution that can be obtained without any splitting.

Lastly, in a somewhat orthogonal approach, Lebbah and Lhomme [17] try to identify cycles in the propagation inside bc3 to avoid slow convergence phenomena. One direction for future research might indeed be to try taking advantage 
of their methods in bcrl.

\section{Conclusion}

Reinforcement learning shows all its potential for difficult problems where no static transversal exists since it realizes a good tradeoff between considering all projections equally (bc3) and gambling on $n$ projections only (standard firstorder methods). The additional cost incurred by the weights update appears negligible, though the same might not hold for their smart initialization. Experimental evidences tend to show that the approach taken with bcrl-j, sophisticated as it is, still incurs a very reasonable overhead in view of its benefits.

Despite its simplicity, the rwa method as we embedded it in bc3 leads to a robust solving algorithm with reliable performances for all kinds of test problems.

There is still room for improvements nonetheless: as is shown on Fig. 7, despite the amelioration obtained with bcrl-j w.r.t. bc3, there still are problems for which many calls to bc3revise do not lead to any domain reduction (most notably, bt24 and ef200). These problems also require a lot of splitting; this may be because bc3revise is itself not a powerful enough algorithm to tighten the domains for these instances (it may be the case when the numerical expressions involved are ill-conditioned).

We have used reinforcement learning to dynamically select the best projections to consider with bc3revise. There is, however, more potential to our method. In particular, we could reuse the principle at the root of bcrl to select both the projection and the pruning method to use with. We would no longer assign weights to projections only, but to pairs of narrowing operators (projection/pruning method). This would lead to consider more than $n^{2}$ operators by creating pairs between the at most $n^{2}$ projections and several pruning methods, such as bc3revise, a unary Newton-Raphson contractor, or other methods, letting the learning procedure dynamically sift the best narrowing operators.

\section{References}

[1] Peter Auer, Nicolò Cesa-Bianchi, Yoav Freund, and Robert E. Schapire. The non-stochastic multi-armed bandit problem. SIAM Journal on Computing, 32(1):48-77, 2002.

[2] Peter Auer, Nicolò Cesa-Bianchi, Yoav Freund, and Robert E. Shapire. Gambling in a rigged casino: the adversarial multi-armed bandit problem. In Procs. of the 36th Annual Symposium on Foundations of Computer Science (FOCS '95), pages 322-331. IEEE Computer Society Press, 1995.

[3] Frédéric Benhamou. Interval constraints, interval propagation. In Panos M. Pardalos and C. A. Floudas, editors, Encyclopedia of Optimization, volume 3, pages 45-48. Kluwer Academic Publishers, 2001. 
[4] Frédéric Benhamou, David McAllester, and Pascal Van Hentenryck. CLP(Intervals) revisited. In Procs. Intl. Symp. on Logic Prog., pages 124138. The MIT Press, 1994.

[5] L. C. W. Dixon and G. P. Szegö. The global optimization problem: an introduction. In L. C. W. Dixon and G. P. Szegö, editors, Towards Global Optimization 2, pages 1-15. North-Holland, 1978.

[6] Iain S. Duff. On algorithms for obtaining a maximum transversal. $A C M$ Transactions on Mathematical Software, 7(3):315-330, September 1981.

[7] Frédéric Goualard. On considering an interval constraint solving algorithm as a free-steering nonlinear gauss-seidel procedure. In Procs. of the 20th Annual ACM Symposium on Applied Computing (Reliable Computation and Applications track), volume 2, pages 1434-1438. The Association for Computing Machinery, Inc., March 2005.

[8] Frédéric Goualard and Christophe Jermann. On the selection of a transversal to solve nonlinear systems with interval arithmetic. In Vassil N. Alexandrov et als., editor, Procs. International Conference on Computational Science 2006, volume 3991 of Lecture Notes in Computer Science, pages 332339. Springer-Verlag, 2006.

[9] Laurent Granvilliers and Gaétan Hains. A conservative scheme for parallel interval narrowing. Information Processing Letters, 74:141-146, 2000.

[10] Eldon R. Hansen and Saumyendra Sengupta. Bounding solutions of systems of equations using interval analysis. BIT, 21:203-211, 1981.

[11] Stefan Herbort and Dietmar Ratz. Improving the efficiency of a nonlinearsystem-solver using a componentwise newton method. Research report 2/1997, Institut für Angewandte Mathematik, Universität Karslruhe (TH), 1997.

[12] Timothy J. Hickey, Qun Ju, and Maarten H. Van Emden. Interval arithmetic: from principles to implementation. J. ACM, 48(5):1038-1068, September 2001.

[13] IEEE. IEEE standard for binary floating-point arithmetic. Technical Report IEEE Std 754-1985, Institute of Electrical and Electronics Engineers, 1985. Reaffirmed 1990.

[14] INRIA project COPRIN: Contraintes, OPtimisation, Résolution par INtervalles. The COPRIN examples page. Web page at http://www-sop.inria.fr/coprin/logiciels/ALIAS/Benches/ benches.html.

[15] Ralph Baker Kearfott, Chenyi Hu, and Manuel Novoa III. A review of preconditioners for the interval Gauss-Seidel method. Interval Computations, 1:59-85, 1991. 
[16] Ralph Baker Kearfott and Xiaofa Shi. Optimal preconditioners for interval gauss-seidel methods. In G. Alefeld and A. Frommer, editors, Scientific Computing and Validated Numerics, pages 173-178. Akademie Verlag, 1996.

[17] Yahia Lebbah and Olivier Lhomme. Accelerating filtering techniques for numeric csps. Artificial Intelligence, 139(1):109-132, 2002.

[18] Ladislav Luksan and Jan Vlcek. Sparse and partially separable test problems for unconstrained and equality constrained optimization. Research report V767-98, Institute of Computer Science, Academy of Science of the Czech Republic, December 1998.

[19] Alan K. Mackworth. Consistency in networks of relations. Artificial Intelligence, 1(8):99-118, 1977.

[20] Ramon E. Moore. Interval Analysis. Prentice-Hall, Englewood Cliffs, N. J., 1966 .

[21] Jorge J. Moré and Michel Yves Cosnard. Numerical solutions of nonlinear equations. ACM Transactions on Mathematical Software, 5:64-85, 1979.

[22] Arnold Neumaier. Interval methods for systems of equations, volume 37 of Encyclopedia of Mathematics and its Applications. Cambridge University Press, 1990.

[23] James M. Ortega and Werner C. Rheinboldt. Iterative solutions of nonlinear equations in several variables. Academic Press Inc., 1970.

[24] Helmut Ratschek and Jon Rokne. Interval methods. In Handbook of Global Optimization, pages 751-828. Kluwer Academic, 1995.

[25] D. G. Sotiropoulos, J. A. Nikas, and T. N. Grapsa. Improving the efficiency of a polynomial system solver via a reordering technique. In Procs. 4th GRACM Congress on Computational Mechanics, volume III, pages 970976, 2002.

[26] Richard Sutton and Andrew Barto. Reinforcement learning: an introduction. MIT Press, 1998. 